\title{
Overview of uniportal video-assisted thoracic surgery (VATS): past and present
}

\author{
J. Matthew Reinersman ${ }^{1}$, Eliseo Passera ${ }^{2}$, Gaetano Rocco ${ }^{3}$ \\ ${ }^{1}$ Department of Surgery, Division of Thoracic and Cardiovascular Surgery, University of Oklahoma Health Sciences Center, Oklahoma City, \\ Oklahoma, USA; ${ }^{2}$ Department of Thoracic Surgery, Humanitas Gavazzeni Institute, Bergamo, Italy; ${ }^{3}$ Department of Thoracic Surgery and \\ Oncology, Division of Thoracic Surgery, Istituto Nazionale Tumori, Pascale Foundation, IRCCS, Naples, Italy \\ Correspondence to: Gaetano Rocco, MD, FRCSEd, FETCS. Department of Thoracic Surgery and Oncology, Division of Thoracic Surgery, Istituto \\ Nazionale Tumori, Fondazione Pascale, IRCCS, Via Mariano Semmola 81, 80131 Naples, Italy. Email: gaetanorocco60@gmail.com.
}

\begin{abstract}
Single incision video-assisted thoracic surgery (VATS), better known as uniportal VATS, has taken the world of thoracic surgery by storm over the previous few years. Through advances in techniques and technology, surgeons have been able to perform increasingly complex thoracic procedures utilizing a single small incision, hence avoiding the inherent morbidity of the standard open thoracotomy. This was a natural extension of what most recognize as the standard of care for early stage lung cancer, the VATS lobectomy, generally performed through a three- or four-incision technique. Improved camera optics have allowed the use of smaller cameras, making the uniportal approach technically easier. Improvement in articulating staplers and the development of other roticulator instruments have also aided working through a small single access point. The uniportal technique further brings the operative fulcrum inside the chest cavity, enabling better visualization, and creates working conditions similar to the open thoracotomy. Currently, uniportal VATS is being used for minor thoracic procedures and lung resections up to complex thoracic procedures typically requiring open approaches, such as chest wall resections, pneumonectomy, and bronchoplastic and pulmonary artery sleeve resections. Uniportal VATS is a clear advance in the field of general thoracic surgery and provides but a glimpse into the untold future.
\end{abstract}

Keywords: Uniportal video-assisted thoracic surgery (VATS); single-incision thoracoscopic surgery; minimally invasive surgery

Submitted Nov 02, 2015. Accepted for publication Mar 02, 2016.

doi: $10.21037 /$ acs.2016.03.08

View this article at: http://dx.doi.org/10.21037/acs.2016.03.08

\section{Introduction}

The evolution of minimally invasive surgery is defined by overlapping epochs. However, this ever-changing landscape is a relatively young field, initially limited by safety, but is now experiencing a rapid growth. Once safety was established utilizing open approaches to operative interventions, surgeons have been seeking methods to decrease the trauma inherent to any operation. In the arena of thoracic surgery, the recent past and present are dominated by the evolution of video-assisted thoracic surgery (VATS) techniques. Therefore we passed through open surgery with a single large incision to a minimally invasive surgery with three-, then two- and lastly singleport VATS. Through the innovation of many surgeons, we are now at the point where major anatomic resections are performed through single incisions of only 2.5 to $5 \mathrm{~cm}$, the uniportal VATS approach. In 2016, it is the time to confer an identity to uniportal VATS with a proper acronym: UniVATS. Thoracic endoscopic single-incision surgery is a very exciting new modality in the field of minimal access surgery which further reduces the scars of standard VATS and works towards the hypothetical climax of scarless surgery. This perspective piece will discuss the past and present of uniportal VATS and make a few prognostications on the future. 


\section{Development of uniportal VATS}

As with the development of many techniques, looking to the past can build a bridge to the future. In 1910, Jacobaeus reported inserting an ureteroscope into the thoracic cavity to inspect the pleura (1). He subsequently published a series of these cases and utilized a second incision to perform pneumolysin for pneumothorax therapy for tuberculosis. Singer in 1924 utilized a specialized thoracoscope through which multiple instruments could be passed, essentially the first uni-VATS. However, these thoracoscopic techniques were abandoned with the advent of medical therapy for tuberculosis (1).

Uni-VATS technique was first described by the senior author (GR) in 2004 (2). He initially reported on his use of the uniportal technique for wedge resections either for diagnosis of interstitial lung disease or for treatment of primary spontaneous pneumothoraces. This series described the method as an ideal way to approach the thoracic cavity to reproduce the open technique. Through a single port, the fulcrum is moved to inside the chest through the introduction of articulating instruments. The approach requires these articulating instruments to avoid mutual interference. This is different than the standard 3 port VATS because it develops along a sagittal plane rather than a latero-lateral one. A single incision 2-2.5 cm long in the sixth intercostal space along the posterior axillary line was the initial standard approach for resection for diagnostic purposes. The incision is positioned along the midscapular line for pathology in the upper lobes or apical segments of the lower lobes or to perform a pleurodesis. This initial experience of 15 patients demonstrated the safety and utility of the uniportal approach to pulmonary resections.

Soon after this, the same group published on utilizing the technique for pleurodesis for pneumothorax. A comparison between the standard three-port VATS and uniportal VATS for spontaneous pneumothorax was performed relating the efficacy of the uniportal approach (3). Since then, the technique has become popular worldwide and is nowadays part of routine practice in thoracic surgery. The early experience with uni-VATS could be attributed to the development of new optics and articulating instruments developed by the pioneers.

Rocco et al. have now published on a series of over 10 years with this technique, performed in 644 patients (4). Indications for the uni-VATS in this series were varied, including pleural effusion or biopsy in $51 \%$, wedge resection in $29 \%$ and a myriad of other indications including sympathectomy, pericardial window, evacuation of hemothorax and mediastinal masses, thus demonstrating the versatile nature of the technique.

\section{Technical notes on uniportal VATS}

In recent years, major advancements towards standardization of uni-VATS have allowed for the procedure to be considered feasible, safe and reproducible (2).

The details of the procedure have been extensively described in the literature (5). A brief summary of a few technical details comprising uni-VATS are listed below:

(I) Length of incision: a single incision less than or equal to $2.5 \mathrm{~cm}$ (no further dissection of intercostal space, no retractors) represents the standard for uni-VATS. In fact, only one $\mathrm{cm}$ incisions are needed for undetermined pleural effusions and thoracic sympathectomies, whereas, for anatomical lung resections, usually $3-5 \mathrm{~cm}$ incisions suffice. Accordingly, uni-VATS for diagnostic and minor therapeutic procedures (including wedge resections) can be performed with a single $2-2.5 \mathrm{~cm}$ port incision. Major pulmonary resections require slightly larger incisions, mostly to retrieve the specimen from the pleural cavity;

(II) Location of incision and approach: placement of the incision should be versatile early in the learning curve so as to face the target lesion. At the top of the learning curve, surgeons can perform uni-VATS for the majority of lesions throughout the chest as well as anatomic lung resections and other complex resections through the fourth and the sixth intercostal spaces, along the midaxillary line (Table 1). Indeed, the placement of the incision is of paramount importance to guarantee the success of the procedure. Uni-VATS has also been described through subxiphoid, trans-subcostal, transaxillary, transsternal, transdiaphrammatic and transcervical approaches;

(III) Type of optics: rigid, flexible, coaxial cable, chip on tip and needlescopic are all various options described;

(IV) Type of instrumentation: straight, curved, articulating, flexible and pre-bent;

(V) Choice of anesthetic setting: general anesthesia is the standard, however many centers have described performing uni-VATS on sedated or awake patients. 


\begin{tabular}{|c|c|}
\hline Problem & Solution \\
\hline Damage to light fiber of conventional thoracoscopy & Use of optic with coaxial light fiber \\
\hline Loss of triangulation & $\begin{array}{l}\text { Use of articulating/prebent instruments/curved adapted instruments with } \\
\text { proximal and distal articulation }\end{array}$ \\
\hline Lack of exposure & $\begin{array}{l}\text { Tilt table posteriorly to view hilum; anteriorly for lymph node dissection; } \\
\text { "puppetry" traction of target area by endograsp }\end{array}$ \\
\hline Difficulty in movements of instruments & $\begin{array}{l}\text { Slightly larger incision like that of } 25 \mathrm{~mm} \text { instead of } 15 \mathrm{~mm} \text { improves movement } \\
\text { ensuring a sufficient distance between the port site and the target area }\end{array}$ \\
\hline
\end{tabular}

\section{Widespread application}

The second major milestone in the use of uniportal VATS techniques was the first uniportal VATS lobectomy. Upon visiting the VATS program at Duke University employing two incision VATS lobectomy, Gonzales-Rivas ventured upon utilizing the uniportal technique to perform a lobectomy. In 2011, he published this first case a uniportal VATS lobectomy (6). His technique includes a $4-5 \mathrm{~cm}$ incision at the fifth intercostal space in the anterior axillary line.

He has since published his two year experience with uniportal VATS lobectomy, including 102 patients, from June 2010 to July 2012 (7). This expanded series included two bilobectomies and five pneumonectomies as well as standard lobectomies. In all, five cases required conversion: three to open thoracotomy and two to 2-port VATS. Interestingly, his series was performed utilizing standard VATS instruments and a $10-\mathrm{mm} 30$ camera with no trocars. Of the 92 patients included in the analysis, the median length of chest tube duration was 2 days and the median length of hospital stay was three days. Mean surgical time was 154.1 minutes. There were complications in 14 patients with no mortality.

This technique in skilled hands was certified to be safe with excellent postoperative outcomes. Subsequently he has utilized this approach for even more complex resections: segmentectomy in 2012, pneumonectomy in 2012, bronchoplastic procedure in 2013, chest wall resection in 2013 and pulmonary artery reconstruction in 2013 (8-12).

Uniportal VATS, particularly lobectomy, has generated a large amount of interest throughout the international community. The initial response to Gonzalez-Rivas' work was that this was the work of an exceptional surgeon, unable to be duplicated elsewhere. This subsequently has been proven wrong by the rapid adoption of this technique across the world. Multiple reports over the last few years confirm this, particularly in Asia and Europe $(13,14)$.

In the past year have now come reports of the subxiphoid uniportal approach. Suda and colleagues first reported their center's experience with a single subxiphoid incision to perform a thymectomy (15). Following this success they applied the approach to access bilateral pleural spaces to perform pulmonary metastasectomy (16). Closely following this report, Liu and colleagues reported their experience with uniportal subxiphoid VATS lung resections, particularly uniportal subxiphoid lobectomy (17). These novel applications of the uniportal technique again demonstrate its versatility and ease of use.

\section{Outcomes}

The main outcome advantage described by advocates of uniportal VATS is decreased pain and paresthesia. The use of only a single incision means less access trauma and only one intercostal space and therefore only one intercostal nerve is likely to be stretched, providing only a single distribution of pain. This theoretically results in less short term and long term pain. Multiple groups have reported on their experience (Table 2).

Jutley and colleagues in 2005 first reported data on pain after uniportal VATS, early in its use (3). This single 


\begin{tabular}{|c|c|}
\hline Outcome & Uniportal VATS and conventional three port VATS \\
\hline Cosmetic result & Better in uniportal VATS (18) \\
\hline Costs & Extra costs by uniportal VATS (could be compensated by shorter hospital stay) (20) \\
\hline Hospital stay & No differences in most published studies \\
\hline Pain & Uniportal VATS may offer improved pain scores (3) \\
\hline Safety and feasibility & No differences (4) \\
\hline Indications & No differences (usually not suitable for complex procedures) \\
\hline Modality of anesthesia & Awake surgery is more manageable in uniportal VATS (21) \\
\hline Postoperative complications & No differences (18) \\
\hline
\end{tabular}

surgeon series compared the use of uniportal VATS (16 patients) to conventional three-port procedures (nineteen patients) for primary spontaneous pneumothorax. There was no difference between the groups in terms of age, spirometry, tissue resected, drainage time and inpatient stay. The uniportal group did have a lower median pain score on the visual analogue scale compared to the three-port technique $(\mathrm{P}=0.06)$. Three-port VATS also had a higher residual pain score (0.5) compared to uniportal VATS $(0.3)$. Further, eighty-six percent of uniportal patients reported no long term neurologic symptoms. The remaining patients experienced only mild 'numbness' or 'swelling'. However, in the three-port group, $42 \%$ reported no symptoms. A similar number experienced 'numbness'. Two females described sexual dysfunction due to altered breast sensitivity. Seventeen percent (2/12) reported 'pins and needles'.

Tamura et al. from Japan retrospectively compared their results of 37 patients in total, 18 3-port VATS operations and 19 single incision VATS (19). A visual analog scale was utilized to assess pain scores and was recorded on postoperative days $0,1,3,7$ and 14 . Pain scores were significantly higher in patients who underwent standard 3 -port VATS on postoperative days 0, 1 and 3 than those who underwent uniportal VATS. There was no difference in length of chest tube duration, duration of surgery or hospital stay. The uniportal group did also report higher satisfaction scores, although this was not statistically significant.

A prospective study by Mier and colleagues presented data from 20 patients also undergoing standard 3-port VATS versus single incision VATS (22). They also compared differences in hospital length of stay, duration of chest tube drainage and postoperative pain. This study was performed in a prospective manner and the groups were also matched according to age and sex. No differences were noted in mean pleural drainage or length of hospitalization. Once again using the mean visual analog scale, pain scores were significantly better for patients undergoing single incision VATS $(4.4 \pm 1.7$ for single incision and $6.2 \pm 1.4$ for 3 port VATS; $\mathrm{P}=0.035$ ).

Importantly, two studies standout displaying the safety of the uniportal technique. Rocco published his 10 year experience of over 644 patients with this technique (4). Mortality was $0.6 \%$. Conversion to 2 - or 3-port VATS or mini-thoracotomy was only $3.7 \%$, which was most often due to incomplete lung collapse (92\%). Major postoperative morbidity was only $2.8 \%$. Uniportal VATS was utilized in one third of all operations during this time frame. The other major study is Gonzalez-Rivas' report detailing his two years of uniportal VATS lobectomy in over 100 patients with zero mortality (7).

A secondary major concern raised regarding the uniportal VATS approach was the potentially increased surgical costs due to the use of roticulating disposable instruments. However, a study by Salati and colleagues compared these factors in 51 consecutive patients undergoing either threeport VATS (23 patients) or uniportal VATS (28 patients) for the management of primary spontaneous pneumothorax (20). 
They found that the costs were comparable between the two groups for surgical materials $(\mathrm{P}=0.69)$ and operating room costs $(\mathrm{P}=0.67)$. However, the patients in the uniportal group had significantly shorter hospital stays (3.8 vs. 4.9 days, $\mathrm{P}=0.03$ ) which resulted in an overall significant reduction in postoperative costs for patients undergoing the uniportal technique $(\mathrm{P}=0.03)$.

\section{Future applications}

Currently, single incision systems are in practice with the Intuitive da Vinci robotic system but these have not been approved for thoracic surgical procedures. Elsewhere on the horizon are smaller cameras with improved optics, flexible thoracoscopes, smaller staples and improved energy devices. These will all be necessary to continue to advance our specialty. Another interesting technology is remote wireless cameras, able to be inserted into the thoracic cavity to provide alternative viewing angles without a cumbersome thoracoscope and related cords. Alternatively, other instruments could also be wireless and anchored externally with magnetic retractors, freeing up the access incision.

The field of video-assisted thoracic surgery continues to advance. The advent of the uniportal technique has added a versatile tool for the thoracic surgeon. The arena of minimally invasive surgery is a relatively young field, with many technological advances still to make an impact in the operating room. Moore's law, named after Gordon Moore, the founder of Intel, states that the number of transistors in a circuit doubles every two years. This "law" has often been applied to describe other areas of technology as well. Advances in optics, including $3 \mathrm{D}$ cameras, ultrahigh definition monitors and 'retina' displays, and robotic technologies will all have a dramatic impact on our ability to continue to offer patients safer surgery with less trauma.

\section{Conclusions}

Uni-VATS is clearly more than a passing fad in the world of thoracic surgery. It offers a valid alternative to conventional VATS techniques and may afford improved postoperative pain and long-term advantages in pain and paresthesia. In the era of managed care and patient satisfaction ratings, the ability to offer patients a safe, oncologically effective, single small incision surgery is a clear advantage (23).

With minimal access surgery changing at a rapid pace, only longer follow-up and randomized controlled studies will predict whether uni-VATS represents the standard approach in thoracic surgery or whether will be designed to perform only selected procedures to be carried out in selected centers.

We are confident that technology will support uni-VATS in the future, so as to facilitate this procedure (i.e., flexible thoracoscopes, smaller instrumentation, new devices for sealing vessels or for targeting nodules and single-armed robotic devices). We should, however, avoid the risk of pursuing uni-VATS too quickly by emphasizing surgical talent at the expense of patient safety and oncological completeness.

\section{Acknowledgements}

None.

\section{Footnote}

Conflicts of Interest: The authors have no conflicts of interest to declare.

\section{References}

1. Moisiuc FV, Colt HG. Thoracoscopy: origins revisited. Respiration 2007;74:344-55.

2. Rocco G, Martin-Ucar A, Passera E. Uniportal VATS wedge pulmonary resections. Ann Thorac Surg 2004;77:726-8.

3. Jutley RS, Khalil MW, Rocco G. Uniportal vs standard three-port VATS technique for spontaneous pneumothorax: comparison of post-operative pain and residual paraesthesia. Eur J Cardiothorac Surg 2005;28:43-6.

4. Rocco G, Martucci N, La Manna C, et al. Ten-year experience on 644 patients undergoing single-port (uniportal) video-assisted thoracoscopic surgery. Ann Thorac Surg 2013;96:434-8.

5. Rocco G. Single-Port Video-Assisted Thoracic Surgery (Uniportal) in the Routine General Thoracic Surgical Practice. Winter 2009;14:326-35.

6. Gonzalez D, Paradela M, Garcia J, et al. Single-port videoassisted thoracoscopic lobectomy. Interact Cardiovasc Thorac Surg 2011;12:514-5.

7. Gonzalez-Rivas D, Paradela M, Fernandez R, et al. Uniportal video-assisted thoracoscopic lobectomy: two years of experience. Ann Thorac Surg 2013;95:426-32.

8. Gonzalez-Rivas D, Fieira E, Mendez L, et al. Single-port video-assisted thoracoscopic anatomic segmentectomy 
and right upper lobectomy. Eur J Cardiothorac Surg 2012;42:e169-71.

9. Gonzalez-Rivas D, de la Torre M, Fernandez R, et al. Video: Single-incision video-assisted thoracoscopic right pneumonectomy. Surg Endosc 2012;26:2078-9.

10. Gonzalez-Rivas D, Fernandez R, Fieira E, et al. Uniportal video-assisted thoracoscopic bronchial sleeve lobectomy: first report. J Thorac Cardiovasc Surg 2013;145:1676-7.

11. Gonzalez-Rivas D, Fernandez R, Fieira E, et al. Singleincision thoracoscopic right upper lobectomy with chest wall resection by posterior approach. Innovations (Phila) 2013;8:70-2.

12. Gonzalez-Rivas D, Delgado M, Fieira E, et al. Single-port video-assisted thoracoscopic lobectomy with pulmonary artery reconstruction. Interact Cardiovasc Thorac Surg 2013;17:889-91.

13. Wang BY, Tu CC, Liu CY, et al. Single-incision thoracoscopic lobectomy and segmentectomy with radical lymph node dissection. Ann Thorac Surg 2013;96:977-82.

14. Ismail M, Helmig M, Swierzy M, et al. Uniportal VATS: the first German experience. J Thorac Dis 2014;6:S650-5.

15. Suda T, Sugimura H, Tochii D, et al. Single-port thymectomy through an infrasternal approach. Ann Thorac Surg 2012;93:334-6.

16. Suda T, Ashikari S, Tochii S, et al. Single-incision subxiphoid approach for bilateral metastasectomy. Ann Thorac Surg 2014;97:718-9.

17. Liu CC, Wang BY, Shih CS, et al. Subxyphoid single-

Cite this article as: Reinersman JM, Passera E, Gaetano Rocco G. Overview of uniportal video-assisted thoracic surgery (VATS): past and present. Ann Cardiothorac Surg 2016;5(2):112-117. doi: 10.21037/acs.2016.03.08 incision thoracoscopic pulmonary metastasectomy. Thorac Cancer 2015;6:230-2.

18. Yang HC, Cho S, Jheon S. Single-incision thoracoscopic surgery for primary spontaneous pneumothorax using the SILS port compared with conventional three-port surgery. Surg Endosc 2013;27:139-45.

19. Tamura M, Shimizu Y, Hashizume Y. Pain following thoracoscopic surgery: retrospective analysis between single-incision and three-port video-assisted thoracoscopic surgery. J Cardiothorac Surg 2013;8:153.

20. Salati M, Brunelli A, Xiumè F, et al. Uniportal videoassisted thoracic surgery for primary spontaneous pneumothorax: clinical and economic analysis in comparison to the traditional approach. Interact Cardiovasc Thorac Surg 2008;7:63-6.

21. Rocco G, La Rocca A, Martucci N, et al. Awake singleaccess (uniportal) video-assisted thoracoscopic surgery for spontaneous pneumothorax. J Thorac Cardiovasc Surg 2011;142:944-5.

22. Mier JM, Chavarin A, Izquierdo-Vidal C, et al. A prospective study comparing three-port video-assisted thoracoscopy with the single-incision laparoscopic surgery (SILS) port and instruments for the video thoracoscopic approach: a pilot study. Surg Endosc 2013;27:2557-60.

23. Rocco G. One-port (uniportal) video-assisted thoracic surgical resections--a clear advance. J Thorac Cardiovasc Surg 2012;144:S27-31. 